\title{
Do We Share a Language? Communitarism and Its Challenges
}

\author{
Matej Drobňák*
}

Received: 20 July 2018 / Accepted: 5 December 2018

\begin{abstract}
The idea that natural languages are shared by speakers within linguistic communities is often taken for granted. Several philosophers even take the notion of shared language as fundamental and that allows them to use it in further explanations. However, to justify the claim that speakers share a language, it should be possible to demarcate the shared language somehow. In this paper, I discuss: A) the explanatory role which the notion of shared language can play, and B) a strategy for demarcating shared languages from within the linguistic production of speakers. The aim of this paper is to show that the indeterminate nature of meaning in natural languages problematizes the intuitive idea of natural languages as shared.
\end{abstract}

Keywords: Communitarism; indeterminacy of meaning; metasemantics; pragmatics; shared language.

\section{Introduction}

We often take the notion of shared language for granted. We talk about English-speaking countries or German grammar and at the New Year we resolve to improve our Spanish or to learn yet another language. To see

* University of Hradec Králové

- Department of Philosophy and Social Sciences, Philosophical Faculty, University of Hradec Králové, Rokitanského 62, 50003 Hradec Králové, Czech Republic

\matej.drobnak@gmail.com

(C) The Author. Journal compilation (c) The Editorial Board, Organon F.

This article is distributed under the terms of the Creative Commons Attribution-NonCommercial 4.0 International Public License (CC BY-NC 4.0). 
a natural language as something what we can (and do) share with other speakers is very intuitive for lay persons as well as for philosophers.

In this paper, I use the label 'communitarism' for those philosophical approaches to natural languages that take the notion of shared language as fundamental. For example, Lewis (1969), Brandom (1994, 2000), and Peregrin $(2014 \mathrm{a}, 2014 \mathrm{~b})$ argue that a shared language is an outcome of some intra-group processes and Borg (2004) argues that the sharing of natural languages is an outcome of innate language modules. ${ }^{1}$ In general, proponents of communitarism believe that natural languages are shared in a sense that members of a community share one set of meaningful expressions and syntactic rules and that speakers play an important role in maintaining shared languages by using them in communication.

However, as Davidson $(1986,1994)$ noted, if we look at how communication works, we can notice that the way we use sentences or expressions is not always in accordance with their pre-learnt, and thus shared, meanings. In other words, the linguistic production of speakers exhibits variability. This variability is caused by the fact that what a speaker means by uttering a sentence depends partially on her general knowledge and beliefs as well as on the broader circumstances of the conversation in which the sentence is uttered.

Many communitarists admit that the notion of shared language alone does not suffice to explain what makes communication successful. Because of that Lewis $(1969,1979)$ and Brandom $(1994,2000)$ stick to the notion of scorekeeping in language games, and Borg (2004) sticks to the distinction between semantic and pragmatic features of natural language processing.

However, if communication includes processes beyond the simple application of a shared language, the question arises what criteria of individuation should be used for the demarcation of shared languages from within the variable linguistic production of speakers. If communitarists believe that speakers within a community share a language and maintain it over time, then it should be possible to track and demarcate the shared language (the shared set of meaningful expressions and syntactic rules) from their linguistic production.

1 Other philosophers, such as Dummett $(1986,1994)$ or Weiss $(2010)$, use the notion of shared language as an important part of their argumentation. 
In this paper, I discuss one of the most influential strategies which communitarists can adopt to demarcate shared languages - the strategy for coherently maintaining the intuitive idea of natural languages as shared and the idea that the linguistic production of speakers exhibits variability. In short, a communitarist can claim that not all the aspects of the linguistic production of speakers are relevant for demarcating what the shared language of a community is. ${ }^{2}$ One way to distinguish between relevant and irrelevant aspects of linguistic production is to stick to some version of semantic-pragmatic distinction. In this paper, I discuss the currently predominant version of this distinction - minimal semantics as advocated by Borg (2004, 2012).

The aim of this paper is to show that the strategy faces serious problems. The problems lie in the fact that the meanings of expressions in natural languages are indeterminate, as well as in its two-step model of communication and understanding, which is currently criticized on empirical grounds (Cosentino et al. 2017).

In the first part of the paper, I will discuss why a coherent view of natural languages should take into consideration the linguistic production of speakers, as well as how the variability of linguistic production challenges the intuitive idea of natural languages as shared. In the second part of the paper, I will present the strategy mentioned above in detail.

I believe that the intuitive idea of natural languages as shared is so pervasive that it is often accepted without explicit reflection. Nevertheless, the aim of the paper is not to argue that we should abandon the notion of shared language, but to point out weaknesses of the strategy for the demarcation of shared languages and to open up a discussion about future improvement. Any alternative to communitarism currently under discussion

2 In some sense, we can say that the strategy is an allusion to Chomsky (1965) and his distinction between competence and performance, because only some performances (actual acts of uttering a sentence) are acceptable as relevant data for the demarcation of shared languages. Note that the competence-performance distinction has been heavily criticized (Labov 1971, 468; Noonan 1999, 21) for its arbitrary preference of some data over others. Thus, if communitarists rely on the restrictions on "performance," then similar objections should apply to them as well. 
brings more problems than solutions and their prospects for providing a coherent view of natural languages are, at least for now, poor. ${ }^{3}$

\section{Common ground}

I start from a simple assumption: any philosophical account of natural languages should take into consideration how speakers understand expressions and sentences and, subsequently, how they use them in communication. The linguistic production of speakers, as an outcome of their competence, should be of some relevance for any philosophical explanation of natural languages simply because natural languages are those languages which developed naturally within communities of speakers and are used by those speakers in communication.

On the other hand, it also sounds intuitively acceptable that linguists, semanticists, or philosophers of language should abstract from the actual linguistic practices of speakers when providing explanations of how natural languages work. There are many reasons for abstracting - including the defectiveness and sloppiness of the actual linguistic production of ordinary speakers. Despite that, abstractions cannot be completely arbitrary. There must be some connection between the results of abstractions and the linguistic production of speakers. Otherwise there would be no justification that those results fit a particular natural language. ${ }^{4}$ In other words, there

3 Semantic holism as a version of an individualistic approach based on the notion of idiolects is an alternative (Rapaport 2000, 2003; Pollock 2014). See (Drobňák 2018) for a discussion of why Quine (1960) is a proponent of an approach which relies on the notion of idiolects as well. Another alternative could be Ludlow (2014) and his idea of microlanguages.

4 Such a result of an abstraction can be, for example, a semantic model of a language. A semantic model represents a natural language by means of a formal language. Formal languages abstract from natural languages by interpreting (hidden) structures of sentences of a natural language as precise and well-defined structures of a formal language. I believe that formalization of natural languages can help us to recognize some reasons for the variability of linguistic production, e.g. to recognize specific syntactic features of indexical expressions. But it does not take into account many other reasons, e.g. the role of the intentions of speakers in the variability of 
must be some criteria of individuation for shared languages which tell us how to abstract from the linguistic production of speakers.

Because of that, the idea that linguists, semanticists, or philosophers of language abstract from some aspects of the linguistic production of speakers sounds more reasonable. The question of which aspects are relevant and which should be overlooked is then decided by criteria of individuation. In such a case, the actual linguistic production of speakers is considered to be a reliable source of data about natural languages, but we are finical in delimiting which aspects of the linguistic production count as a reliable source.

To sum up, any coherent view of natural languages should somehow reflect upon the actual linguistic practice; otherwise it is not clear what makes it about a natural language. In particular, since the actual linguistic production is performed by speakers in communication, any coherent view of natural languages should be able to give compatible answers to three questions:

a) What natural languages are?

b) What role particular speakers have in maintaining meaningful expressions in natural languages?

c) How communication relates to the previous questions?

\subsection{The challenge}

The biggest challenge in providing the answers for a), b), and c) is that the traditional and very intuitive idea of language does not fit very well with the way in which communication works.

The traditional view in semantics or philosophy of language is that language is a set of meaningful expressions and syntactic rules. Such a view of language is implied if the principle of compositionality is accepted. Standard approaches that aim at delivering semantic models of languages assume that the meanings of words and syntactic rules are sufficient for composing the meanings of sentences (usually understood as truth conditions). The lexicon

their linguistic production. The idea will be further discussed in Section 3 under the label of syntactically-triggered context sensitivity. 
of a language, i.e. the set of meaningful expressions, serves as a stock of building blocks for compositionality. If I learn the meaning of an expression, I can use it (together with other expressions and syntactic rules) to compose what is basically an infinite number of sentences. The role of expressions as building blocks for compositionality is facilitated by the fact that the majority of expressions have determinate and context-invariant meanings.

Such a view of languages has a very high explanatory potential. First of all, a language that mostly consists of expressions with one determinate and context-invariant meaning is easy to learn and share. If we assume that different speakers can acquire and share the meanings of expressions (or concepts as their mental representations), we can easily answer all three of the above questions. We can explain what a natural language is by saying that it is the set of meaningful expressions and syntactic rules which is shared by some speakers. By learning the shared language, speakers become competent and maintain the language for subsequent generations. Then, a group of people counts as a linguistic community if and only if almost all its members share the same language. In the same way, we can say that different speakers understand each other because they share the pre-learnt language and their communication is successful because they ascribe the same meanings to the same expressions.

The biggest problem of this approach is that such an explanation of communication does not have much support if we look at how it actually works. Davidson $(1986,1994)$ argues that the way in which understanding is reached shows it to be untenable that all speakers simply assign the same pre-learnt meanings to the same expressions in communication. Such a view of understanding is untenable, because how we understand a sentence may be influenced by contextual cues present in a conversation. Cues can be intentionally incorporated into conversation by a speaker, or they might be a result of the accidental circumstances in which a conversation takes place. As Davidson $(1986,439)$ demonstrates through Donnellan's (1968) use of the sentence 'There's glory for you,' even this sentence can be understood as 'There's a nice knockdown argument for you' if the conditions are right. Because of these conversational shifts in meaning, the linguistic production of speakers exhibits variability. 
However, the point I want to emphasize is not about the explanation of communication. As I mentioned above, communitarists often admit that the explanation of successful communication requires more than just a simple application of a shared language. Rather, the point I want to emphasize is about demarcating the boundaries of shared languages. If our linguistic production (the way we use expressions) is a source of data for demarcating shared languages and, at the same time, there is a realm of linguistic production that exhibits variability, then the variability of the data indicates that expressions do not have determinate and context-invariant meanings and so it problematizes the possibility of demarcating shared languages.

The variability of linguistic production leads Davidson to the conclusion that there is nothing that corresponds to the standard view of language as a set of meaningful expressions and syntactic rules and we should abandon it. He claims that "there is no such thing as a language, not if a language is anything like what many philosophers and linguists have supposed" (Davidson 1986, 446) and he believes that "we must give up the idea of a clearly defined shared structure which language-users acquire and then apply to cases" (Davidson 1986, 446). I agree that the argument is valid, but only under the assumption that all linguistic production is taken to be a relevant source of data about shared languages. If the circumstances of particular conversations influence our linguistic production and all linguistic production is taken to be a relevant source of data about natural languages, then communitarists are losing the demarcation criterion for what counts as a shared language.

Rejecting the assumption that all linguistic production is a reliable source of data about natural languages might help communitarists to avoid Davidson's conclusion, but the variability of linguistic production (as a fact about natural languages and their use) still poses a challenge for them. The challenge for communitarists is to give clear criteria for which aspects of the linguistic production of speakers count as a reliable source of data for demarcating shared languages and which aspects should be considered to be irrelevant. If communitarists want to preserve the notion of shared language, then they have to explain the existence of the variability of linguistic production along with the existence of shared languages. 


\section{Communitarism}

One strategy for avoiding the challenge is to bite the bullet and to accept that the variability of linguistic production shows that the meanings of expressions in natural languages are, in some sense, variable. Biting the bullet does not necessarily mean the loss of the notion of shared language. The idea might be that, even though there are several ways in which an expression is used in communication, the ways are well recognized and shared by speakers within a community. The context-invariant meaning can be explicated as a compound of several contextual values and the shared language as a set of expressions with complex and variable meanings and syntactic rules. Such a view of meaning is sometimes labelled a 'rich meaning approach' but, as far as I know, this approach to meaning and shared languages has not been spelled out in detail so far.

The biggest problem of this strategy is that it is not clear whether meaning understood in this way can be compositional and thus whether creating a semantic model of a language would be possible. Another problem of this approach is that if meanings are complex compounds, acquiring such languages would be much more demanding (and probably almost impossible). Even if this strategy allows communitarists to save the notion of shared language (in the new sense), such a notion of shared language would not be able to play the same explanatory role as the standard notion of shared language was supposed to play - causing new complications and problems that must be solved.

\subsection{Which aspects of linguistic production?}

Another strategy for preserving the intuitive notion of shared language relies on setting a clear boundary between those aspects of linguistic production which are shared by all speakers and those aspects which can vary from speaker to speaker, from conversation to conversation. As stated earlier, the notion of shared language is often taken for granted without explicit reflection, so it is hard to find an explicit proponent of this strategy. However, I believe that the strategy can be naturally linked to Grice's (1957, 1961) distinction between the semantic and pragmatic features of content. I believe that, if asked, many philosophers would stick to an explanation in 
line with the Gricean distinction between semantics and pragmatics-we share a language with regard to semantics and the variability of our linguistic production is caused by pragmatics.

In particular, a proponent of communitarism can claim that only those aspects of our linguistic production which are relevant for semantic features of content serve as a reliable source of data about shared languages. If communitarists can succeed in demarcating which aspects of our linguistic production correspond to the semantic features of content, then they basically succeed in responding to the challenge. Even though this might not be its primary purpose, minimal semantics as advocated by Borg $(2004,2012)$ can serve as a very good background for accomplishing this task.

According to Borg $(2004,2012)$, formal semantics should deal with the literal meaning of sentence-types and expressions. More specifically, formal semantics should provide a model of a language that is able to state what each sentence of a language means, solely on the basis of the syntactic features of sentences and the semantic properties of its constituents (particular expressions of a language). Stating this standardly amounts to stating the truth conditions of sentences. ${ }^{5}$

What matters for semantic operations on a formal account just are the (local) syntactic properties of representations. So, on this kind of picture, grasp of meaning would seem to be in principle amenable to a (Turing-style) computational explanation. If, say, we treat grasp of literal linguistic meaning as the canonical derivation of truth conditions for sentences, for example, along the lines of Larson and Segal 1995, then semantic understanding can

5 According to Borg, this also allows the incorporation of syntactically triggered context-sensitive expressions. Overt context-sensitive expressions (e.g. demonstratives, indexicals, tensed expressions) may count as such. Syntactically triggered context-sensitivity amounts to cases in which context-sensitivity is somehow "built into" an expression. In other words, context-sensitivity is, in such cases, a matter of the syntactic properties of an expression - it is recognized by a hearer automatically just by hearing an expression, regardless of the broader context of a conversation. This sort of context-sensitivity is in striking contrast to different sorts of context-sensitivity such as conversational implicatures, which require knowledge of the context of a conversation in order to be recognized by hearers. 
form part of a genuine language module, for this is clearly a function which is encapsulated and computational. Knowledge of meaning, on this kind of account, consists of knowledge of a proprietary body of information (the lexicon for the language) and knowledge of a set of rules operating only on that information, rules which consist of formal transformations of the data. (Borg 2004, 81)

Knowing the "proprietary body of information" requires knowing how to categorize objects under particular expressions of a language, i.e. knowing which expressions are related to which concepts. Note that, according to Borg, T-sentences map natural language sentences to "Mentalese," so it makes sense to say that concepts are mental representations of meanings and so the categorization of objects under expressions is relevant for the semantic processing of sentences. If this is so, then referential aspects of our linguistic production can provide relevant data for demarcating literal meanings, as referential aspects of our linguistic production indicate how a speaker categorizes objects. ${ }^{6}$

Aspects of our linguistic production, which are not syntactically encoded, are a matter of what can be implied by uttering a sentence and belong to pragmatics. They are irrelevant for the semantic meaningfulness of expressions and syntactic processing of sentences. But most importantly, if minimal semantics is adopted as a background theory for communitarism, then we can say that the aspects of our linguistic production that are not syntactically encoded are irrelevant for the demarcation of shared languages.

More generally, there are two aspects of minimal semantics that make this theory appealing for communitarists:

6 "It would also fall within the purview of the language faculty to calculate the mental representation of the truth-condition for the natural language sentence 'The cat is on the mat,' where what is constructed is a language of thought sentence which exhibits connections to the external world just to the extent that the language of thought expressions out of which it is constructed exhibit such relations (to put it crudely, since CAT hooks up to cats, and MAT hooks up to mats, the truth conditions for the natural language sentence 'the cat is on the mat' turns on how things stand with some cat and some mat)." (Borg 2004, 24, emphasis added) 
a) since the meaning of a sentence is syntactically encoded, it is possible to determine the literal meaning of a sentence (and so to understand its literal meaning) without any information about the circumstances of a conversation. This can be done solely on the basis of information about the literal meaning of lexical units and the syntactic structure of a sentence and this information is accessible by all speakers under all circumstances simply by hearing a sentence; ${ }^{7}$

b) since minimal semantics is closely linked to the modular theory of mind, ${ }^{8}$ models provided by formal semantics are supposed to be models of a specific linguistic module which is responsible for semantic processing. In general, this module is considered to be innate and this gives us a reason to assume that different speakers process the literal meaning of sentences in the same way. In other words, different speakers ascribe the same meanings to the same expressions/sentences.

If a communitarist adopts minimal semantics, she can claim that the aspects of our linguistic production that are related to syntactically encoded truth conditions of sentences serve to demarcate what shared languages are. Since congruence on concepts (categorization of objects) matters for stating the truth conditions of sentences, only the referential aspects of our linguistic production are relevant for the meaningfulness of particular expressions. A language is then a set of meaningful expressions and syntactic rules with regard to the syntactically encoded truth conditions of sentences and referential aspects of the linguistic production of speakers. If we add the assumption that such a language is an outcome of our innate semantic processing module, we can expect all the speakers within a community to share a language. This allows communitarists to save the notion of shared language and use it in further explanations. For example, it can be used to state a demarcation criterion for linguistic communities: what makes a group of

7 "What minimalism specifies is the content a competent language user is guaranteed to be able to recover, given adequate lexical resources" (Borg 2012, 63).

8 Borg (2004) overtly discusses the modular theory advocated by Fodor (1983, 1998, 2000). Another modular approach can be found in (Chomsky 1971, 1975, 1986, 2000). 
speakers a linguistic community is the fact that they all share a language in the aforementioned sense - that they all share semantic processing with regard to the truth conditions of sentences and they agree on the categorization of objects falling under particular expressions.

A modular theory of mind also answers what role particular speakers play in establishing and maintaining a natural language. As syntactic processing is innate, it does not require any special effort. We are all disposed to process sentences syntactically in the same way simply by virtue of being normal human beings. All we need to do is to show our successors which expressions refer to which objects in the world to the extent that they are able to grasp the corresponding concepts. ${ }^{9}$

As long as we agree on which objects fall under 'blood,' 'hands,' 'the room,' etc. in the sentence 'The man over there left the room with blood on his hands,' we can all (semantically) process and understand the sentence in the same way. Without doubt, much more can be implied by uttering the sentence (e.g. that the man is a killer), but minimal semantics allows communitarists to discriminate minimal standards that must be shared by all speakers and it allows communitarists to demarcate natural languages in terms of these minimal shared standards. ${ }^{10}$

\subsection{Communitarism and communication}

If communitarists adopt minimal semantics, the most natural view of communication may be a two-step model: semantic processing first, pragmatic

9 Allowing that the process of "grasping concepts" can be, at least partially, innately driven: "Finally, then, it seems that we might recognize a third way in which to understand what a module is, for we might view a module as a combination of our two previous accounts, so that a cognitive module comprises a proprietary body of information together with a proprietary set of rules or processes operating over that information. Again, both the rules and the representations they operate on are usually thought to be given innately; thus we have a model of a module as an innate and dedicated cognitive processor" (Borg 2004, 76).

10 This is not to say that Borg herself is a proponent of this view. My only assumption in this paper is that her view can be used to demarcate shared languages in such a way and that such a view might be intuitively appealing for many communitarists. 
processing second. ${ }^{11}$ When a hearer hears a sentence, she first processes it unconsciously via a semantic module. The result is that she understands what a sentence means (semantic understanding). In the next step, all the pragmatic information about the speaker and other circumstances intervenes and the hearer comes up with an interpretation of what the speaker might want to imply by uttering this sentence (pragmatic understanding). The reason why this approach to communication might be appealing for communitarists is that according to this view the notion of shared language is necessary for the explanation of how communication works. According to this proposal, pragmatic processing is only possible with background semantic processing. To reach a pragmatic understanding, which is usually what we care about in communication, a hearer must be "on the same page" as a speaker with regard to the literal meanings of sentences. This requires that they both share a language with regard to the truth conditions of sentences and categorization of objects under particular expressions. If this is not the case, then the initial data required for pragmatic processing might lead the hearer astray.

To sum up, minimal semantics a) is able to preserve the notion of shared language by delimiting truth-conditional and referential aspects of linguistic production as relevant for the demarcation of shared languages and b) relies on the notion of shared language in the explanation of how communication works by postulating a congruent semantic understanding as a precondition for pragmatic understanding.

11 This is the view held by Borg as well. In general, Borg does not think that formal semantics should be able to explain how communication works, and she does not aim at giving such an explanation. But by setting minimal semantics into a modular theory of mind, she sets the idea of minimal semantics into a broader view of how semantic and pragmatic aspects of understanding relate to each other. And this relation indicates a two-step model: "On the one hand, then, semantic knowledge is important and special — without it we would be robbed of the ability to interpret the meanings of words and sentences and thus linguistic communication would be impossible. Yet, from another perspective, semantic knowledge is quite unimportant and peripheral - without all the other kinds of knowledge we have, semantic understanding would be pretty much worthless" (Borg 2004, 263).

Organon F 26 (4) 2019: 572-596 


\section{Problems of the strategy}

There are two problems for communitarists adopting this strategy. The first problem is related to the minimal standards that must be globally shared by all speakers. The second problem is related to the two-step model of communication.

\subsection{Global sharing}

The requirement of a shared language with regard to the sharing of the meanings of particular expressions seems to be too strong to expect. The problem is that the meanings of many expressions in natural languages are not fully determinate and context-invariant.

The point about context-invariance can be demonstrated through examples of free pragmatic enrichment. On the basis of what a hearer might know or find out during conversations, her understanding of the verb 'stop' in the sentence 'The policeman stopped the car' may vary, depending on whether the policeman was standing in the road, sitting in the car, or chasing the car. ${ }^{12}$ As Recanati argues, the circumstances of a conversation in such cases influence not only pragmatic aspects of content (i.e. what is implicated by the sentence) but also the meaning of the sentence, because each way of stopping the car (by issuing a proper signal, by depressing the brake pedal, or by firing a warning shot) is related to different truth conditions.

In this paper, I will put the topic of context-invariance aside and I will focus on the indeterminate nature of meaning in natural languages in detail. The indeterminate nature of meaning in natural languages is often flouted because it is usually taken to be a problem of a small number of expressions only, i.e. vague expressions. Vague expressions share one characteristic feature - objects categorized under them can be ranked on a scale ranging from those which certainly belong in a category to those which certainly do not. Even though there are some uncertain cases, we all have a clear idea of

12 The example is a modification of an example from (Recanati 2004, 2010). The same point could be demonstrated by the example of painted leaves as discussed in (Travis 1997). 
a scale on which we move, i.e. we have well-established and shared criteria of categorization. For example, most people would agree that the percentage of the surface of a head without hair or the density of hair are among the relevant criteria for 'bald.' Different speakers may diverge on how they actually set thresholds, but it does not necessarily mean that their concepts diverge as well, i.e. that they use different criteria for the categorization of objects.

I believe that the indeterminate nature of meaning in natural languages is much more widespread. First of all, it may concern any expression in a natural language -including those that are not standardly understood as vague. Basically, for any expression in a natural language we can find circumstances in which the application of a criterion of categorization is unclear or undecided. The reason is that our linguistic practice is adjusted in accordance with some standard conditions in which we apply criteria of categorization. However, we all experience unusual conditions from time to time. In conditions in which it is not clear which criterion of categorization should be applied the decision is often in the hands of the participants in a conversation. If this is so, then it opens up the possibility that different speakers make different decisions and so they use different criteria of categorization, i.e. they assign different (though probably similar) concepts to one expression. Note that this is not vagueness as it is standardly understood. The problem I am discussing here is that we do not know whether some criteria of categorization are relevant, while in the case of vagueness we know what the relevant criteria are (we know the scale) but we do not know the exact thresholds.

Let us demonstrate this through the case of an expression that is not standardly considered to be vague - 'actor.' Most people would agree that an actor is a person whose profession is acting in films or television and the number of appearances in films or whether acting is their main source of income are among the relevant criteria of categorization. ${ }^{13}$ In 2011, Orlean published an article in The New Yorker about Rin Tin Tin, a movie star from the twenties. He was a real star of those times - he starred in more than 20 films made by Warner Bros. and received the Abraham Lincoln

13 At least, this is a definition of the term provided by the Oxford Dictionary. See: https://en.oxforddictionaries.com/definition/actor. 
humanitarian award and the medal for distinguished service, and the Mayor of New York gave him a key to the city. He was at the peak of his career in 1929 when he received the most votes for the best actor for the Academy Award. The only trouble was that Rin Tin Tin was a German Shepherd and the members of the Academy decided that a dog could not win the prize for the best actor.

Note that the question whether a dog can be an actor is not a case of vagueness. It is not a matter of how we decide to set the thresholds on a standard scale - Rin Tin Tin was the main character of many films and he was paid for his acting. He was even famous for real acting, as opposed to merely appearing on the stage (he was able to build the atmosphere of a scene by his facial expressions and so on). The question was whether being a human being is a relevant criterion for the categorization of objects under 'actor' and there is no vagueness in that; there is no blurred area of problematic cases. And yet, there was no definite answer to this question.

The case can be interpreted in two ways and both of them undermine the idea of minimal shared semantic standards and semantic processing. First, we can say that the meaning of 'actor,' or a corresponding concept, was indeterminate before the voting and it was only after realizing this indeterminacy that different people made it a little more precise. ${ }^{14}$ If this was the case, then it is hard to say what sharing indeterminate meanings/concepts amounts to. How can we say that two speakers shared the same meaning of 'actor' if it was not clear what the meaning was? How can we decide whether a concept possessed by one speaker is the same as a concept possessed by another speaker if it is indeterminate which criteria of categorization are constitutive for the concept? A natural response to this worry would be to say that those speakers possessed similar concepts or that their understanding of the expressions partially overlapped. However, as far as I know, there is no viable theory of concept/meaning similarity currently under discussion. ${ }^{15}$

14 Note that different people made different decisions so if this was the case, then the term became ambiguous. For the voters, 'actor' could include dogs; for members of the Academy it could only include human beings.

15 See (Fodor and Lepore 1999) for a critical evaluation of Churchland's (1986, 1993) notion of meaning similarity. 
Another interpretation of the Rin Tin Tin case is that even before the case it was determinate whether dogs fell under the concept of 'actor' but the question did not arise. ${ }^{16}$ In such a case, the unusual circumstances forced people to compare their understanding of 'actor' with respect to the categorization of dogs. The people who voted for Rin Tin Tin believed that a dog could count as an actor; the members of the Academy were a rather more conservative in their criteria of categorization. The understanding of 'actor' within a linguistic community had been challenged and it uncovered discrepancies between the concepts possessed by different speakers and thus meanings assigned to the same expression. If this was the case, then clearly the idea that all the speakers within a community share a semantic understanding on the level of particular expressions, i.e. ascribe the same meanings to the same expressions, does not have much support.

The idea that expressions of natural languages are indeterminate is not new. Waismann's (1945) idea of open texture goes in the same direction and Gauker (2017), as a current proponent of the idea of open texture, overtly argues that it is problematic to simply assume that we all share the same fully determinate concepts (even though in most cases our criteria of categorization deliver overlapping results). ${ }^{17}$ Wilson (1982) proposes a thought experiment that aims to demonstrate that our criteria of categorization are often influenced by accidental features of situations in which decisions are made. ${ }^{18}$ Ludlow (2014) argues that our criteria of categorization are dynamic, i.e. they can change from conversation to conversation.

The lesson to be learned from the Rin Tin Tin case is that there is never a guarantee that there is a special realm of semantic processing which is

16 This is certainly an oversimplification. At least, it had never received so much attention.

17 However, see (Shapiro 2006) for a critical discussion. Shapiro argues that opentexture should count as a kind of vagueness. As far as I can see, the discussion does not have a winner so far.

18 The thought experiment is about an airplane that has fallen into the jungle. According to Wilson, the decision as to whether a jungle tribe will consider the plane to be a strange house or a strange bird depends on whether they see the plane before its fall or find it after the fall. 
shared by all speakers. Even in a case such as 'actor,' in which we usually assume the congruence of our concepts without any doubts, we can find differences among the members of a linguistic community. ${ }^{19}$ Note that the indeterminacy revealed by unusual circumstances is not the exclusive domain of the term 'actor.' The term 'actor' is not usually considered to be vague or non-standard in any other way. This suggests that this kind of indeterminacy might be a general feature of natural languages. If this is so, then it should not be a problem to find more such cases. To illustrate that this phenomenon is more common than we might think, I will present more examples.

Ludlow (2014) discusses the very similar case of whether Secretariat (a horse) can be an athlete. The discussion over Secretariat followed a very similar pattern to the one over Rin Tin Tin. Sport Illustrated placed Secretariat on the list of the best athletes of the last century. This decision sparked a public debate. Some people defended the choice and some people disagreed. From the linguistic point of view, this discussion can be understood as revealing discrepancies in the criteria for the categorization of objects under the term 'athlete' between different competent English speakers. Similarly, Johnson (2018) discusses in The Conversation how current advances in technology and science problematize our understanding of the term 'meat.' The question that Johnson poses in her article is whether labgrown meat should also be considered to be meat. From the linguistic point of view, this is a question of whether the standard criteria of categorization for the term 'meat' apply to lab-grown meat as well. As in the last two cases, there are people who believe that lab-grown meat counts as meat and there are people who disagree. The changed circumstances (caused by advances in technology) reveal the indeterminacy of a term that was not considered to be indeterminate. It shows how different competent speakers may apply different criteria of categorization without knowing about it until challenged. ${ }^{20}$

19 A similar point leading to a conclusion that decisions about the categorization of particular objects depend on accidental features of particular situations, and so it is hard to expect congruence among all speakers, was raised in (Wilson 1982, 2006). 20 Similarly, we could interpret the discussion over the status of Pluto as a linguistic discussion over the criteria of categorization of the term 'planet.' As in the case 
The most important point, however, is that we can never rule out the possibility that we will stumble upon such indeterminacy or differences in concepts/meanings for any expression in a natural language because we can never assess all the possible circumstances in which an expression can be used. For any expression, there is a possibility that there will be some circumstances which may reveal differences in categorization which have not been noticed before. If this is so, then the assumption that there is a special realm of semantics on the level of particular expressions, which is shared by all speakers, seems at least problematic and deserves more attention by any communitarist adopting this strategy.

\subsection{Two-step model of communication}

The second problem of this strategy is the two-step model of communication. The idea that we first semantically process what we hear and it is only after that that we start pragmatic processing has been undermined by recent empirical research. Werning and Cosentino (2017) and Cosentino et al. (2017) show that free pragmatic enrichment intervenes even during the early stages of the semantic processing of sentences. ${ }^{21}$ More specifically, free pragmatic enrichment helps us to modulate word meanings before we process a sentence semantically. The research was focused on the neurological activity of subjects during the processing of congruent vs. incongruent noun-verb combinations. ${ }^{22}$ More specifically, research teams tested how neurological activity depends on the context of a sentence. A context was presented to subjects as a short story and it served as information necessary for free pragmatic enrichment. Beside other combinations, the researchers also tested how neurological activity changes

of 'meat,' new circumstances have been caused by advances in science. Specifically, by the discovery of other planet-size objects in Kuiper belt.

21 Free pragmatic enrichment is a process in which the semantic understanding of a term is influenced by a context of a conversation before the semantic processing of a sentence is finished. The term 'free pragmatic enrichment' was coined by Recanati (2004, 2010). See the beginning of subsection 4.1 for an example and a short explanation of the term.

22 Cosentino uses funnel-pour as an example of congruent combination and funnelhang (a coat) as an example of incongruent combination.

Organon F 26 (4) 2019: 572-596 
when we combine a congruent context ${ }^{23}$ with congruent and incongruent noun-verb combinations and when we combine an incongruent context ${ }^{24}$ with congruent and incongruent noun-verb combinations.

According to the two-step strategy, subjects should first semantically process the literal meaning of the whole sentence and only then should pragmatic processing take place. If this is so, then we can predict that the context of a sentence should not influence neurological activity related to the semantic processing of particular words (or noun-verb combinations). More specifically, the neurological activity at the time of $400 \mathrm{~ms}$ after hearing a verb (N400 component $)^{25}$ should be the same, regardless of the context. However, the research shows that the neurological activity after hearing a verb is significantly affected in those cases in which a congruent context is followed by an incongruent noun-verb combination and vice versa (incongruent context and congruent noun-verb combination). ${ }^{26}$ In other words, the same noun-verb combination elicits different neurological activity in different contexts. If we assume that the neurological activity (N400 component) corresponds to the contribution of a particular word to the processing of the meaning of a sentence, then the difference shows that the context influences a word's semantic contribution before the semantic processing of a sentence is finished.

23 A context inducing a congruent noun-verb combination. In the case of funnelpour, that would be a context of standard procedures in a chemical laboratory or in wine cellar.

24 A context inducing an incongruent noun-verb combination. In the case of funnelhang (a coat), that would be a context of creative work at an art class.

25 The phenomenon of neurological activity peaking at the time of $400 \mathrm{~ms}$ after semantically oriented stimuli was reported for the first time by Kutas and Hillyard (1980) and confirmed several times after that in different experimental settings (Baggio et al. 2008; Kutas and Hillyard 1984; Kutas et al. 1984; Kutas and Federmeier 2011)

26 Neurological activity was measured by EEG as electrical activity in a specific region of the brain $400 \mathrm{~ms}$ after hearing a word. The influence of context was associated with the difference in electrical activity of the same part of the brain triggered by hearing the same word in different contexts. 
More research has to be done to find out how exactly we should interpret the N400 component, ${ }^{27}$ but all the interpretations currently under discussion hold that it represents the contribution of particular expressions to the semantic processing of a sentence. Differences in neurological activity varying in accordance with different contexts indicate that pragmatic processing takes place even before the semantic processing of a sentence is finished and so it undermines the two-step model of understanding and communication. But if the two-step model of communication is undermined, then the idea of a shared language based on minimal shared standards is problematized as well. There seems to be no special realm of semantic processing on the level of particular expressions which would be shared by all speakers.

\section{Conclusions}

In this paper, I discussed one strategy which communitarists can adopt for coherently maintaining the idea of a natural language as shared and the idea that the linguistic production of speakers exhibits variability in communication. According to the strategy, only some aspects of the linguistic production of speakers are relevant for demarcating a shared language. This strategy can naturally be supported by some version of a semantic-pragmatic distinction if the semantic features of content are considered to be shared by all speakers. The first problem of this strategy is that the meanings of expressions in natural languages are indeterminate and so it is hard to say what sharing meanings of expressions, and thus sharing a language, might amount to. The second problem is its two-step model of communication and understanding, which is currently criticized on empirical grounds.

\section{Acknowledgments}

I would like to thank an anonymous reviewer for helpful suggestions and comments on an earlier version of this paper.

27 See (Baggio and Hagoort 2011); (Brouwer and Hoeks 2013); (Hagoort et al. 2009) for discussion. 


\section{Funding}

Work on this paper was supported by the joint Lead-Agency research grant between the Austrian Science Foundation (FWF) and the Czech Science Foundation (GAČR) "Inferentialism and Collective Intentionality," GF17-33808L.

\section{References}

Baggio, Giosuè, van Lambalgen, Michiel, and Peter Hagoort. 2008. "Computing and Recomputing Discourse Models: An ERP Study." Journal of Memory and Language 59 (1): 36-53. https://doi.org/10.1016/j.jml.2008.02.005

Baggio, Giosuè, and Peter Hagoort. 2011. "The Balance between Memory and Unification in Semantics: A Dynamic Account of the N400." Language and Cognitive Processes 26 (9): 1338-67. https://doi.org/10.1080/01690965.2010.542671

Borg, Emma. 2004. Minimal Semantics. Oxford: Oxford University Press. https://doi.org/10.1093/0199270252.001.0001

Borg, Emma. 2012. Pursuing Meaning. Oxford: Oxford University Press. https://doi.org/10.1093/acprof:oso/9780199588374.001.0001

Brandom, Robert. 1994. Making It Explicit: Reasoning, Representing, and Discursive Commitment. Cambridge, MA: Harvard University Press.

Brandom, Robert. 2000. Articulating Reasons: An Introduction to Inferentialism. Cambridge, MA: Harvard University Press.

Brandom, Robert. 2007. "Inferentialism and Some of Its Challenges." Philosophy and Phenomenological Research 74 (3): 651-76.

https://doi.org/10.1111/j.1933-1592.2007.00044.x

Brouwer, Harm, and John C.J. Hoeks. 2013. "A Time and Place for Language Comprehension: Mapping the N400 and the P600 to a Minimal Cortical Network." Frontiers in Human Neuroscience 7 (758): 1-12. https://doi.org/10.3389/fnhum.2013.00758

Chomsky, Noam. 1965. Aspects of the Theory of Syntax. Cambridge, MA: MIT Press.

Chomsky, Noam. 1971. "Recent Contributions to the Theory of Innate Ideas." In Philosophy of Language, edited by John Searle, 121-29. Oxford: Oxford University Press.

Chomsky, Noam. 1975. Reflections on Language. New York: Pantheon.

Chomsky, Noam. 1986. Knowledge of Language. New York: Praeger.

Chomsky, Noam. 2000. New Horizons in the Study of Language and Mind. Cambridge: Cambridge University Press.

https://doi.org/10.1017/CBO9780511811937 
Churchland, Paul M. 1986. "Some Reductive Strategies in Cognitive Neurobiology." Mind 95 (379): 279-309. https://doi.org/10.1093/mind/XCV.379.279

Churchland, Paul M. 1993. "State-Space Semantics and Meaning Holism." Philosophy and Phenomenological Research 53 (3): 667-72.

https://doi.org/10.2307/2108090

Cosentino, Erica, Giosuè Baggio, Jarmo Kontinen, and Markus Wernig. 2017.

"The Time-Course of Sentence Meaning Composition. N400 Effects of the Interaction between Context-Induced and Lexically Stored Affordances." Frontiers in Human Neuroscience 8 (813): 1-17.

https://doi.org/10.3389/fpsyg.2017.00813

Davidson, Donald. 1986. "A Nice Derangement of Epitaphs." In Truth and Interpretation: Perspectives on the Philosophy of Donald Davidson, edited by Ernest Lepore, 433-46. Cambridge: Blackwell.

Davidson, Donald. 1994. "The Social Aspect of Language." In The Philosophy of Michael Dummett, edited by Brian McGuinness and Gianluigi Oliveri, 1-16. Dordrecht: Kluwer Academic Publishers. https://doi.org/10.1007/978-94-0158336-7_1

Donnellan, Keith. 1968. "Putting Humpty Dumpty Together Again." The Philosophical Review 77 (2): 203-15. https://doi.org/10.2307/2183321

Drobňák, Matej. 2018. "Quine on Shared Language and Linguistic Communities." Philosophia 46 (1): 83-99. https://doi.org/10.1007/s11406-017-9916-y

Dummett, Michael. 1986. "A Nice Derangement of Epitaphs: Some Comments on Davidson and Hacking." In Truth and Interpretation: Perspectives on the Philosophy of Donald Davidson, edited by Ernest Lepore, 459-76. Cambridge: Blackwell.

Dummett, Michael. 1994. "Reply to Davidson: The Social Aspect of Language." In The Philosophy of Michael Dummett, edited by Brian McGuinness and Gianluigi Oliveri, 257-62. Dordrecht: Kluwer Academic Publishers. https://doi.org/10.1007/978-94-015-8336-7_13

Fodor, Jerry. 1983. Modularity of Mind. Cambridge, MA: MIT Press.

Fodor, Jerry. 1998. Concepts: Where Cognitive Science Went Wrong. Oxford: Oxford University Press. https://doi.org/10.1093/0198236360.001.0001

Fodor, Jerry. 2000. The Mind Doesn't Work That Way. Cambridge, MA: MIT Press.

Fodor, Jerry, and Ernest Lepore. 1999. "All at Sea in Semantic Space: Churchland on Meaning Similarity." Journal of Philosophy 96 (8): 381-403. https://doi.org/10.5840/jphil199996818

Gauker, Christopher. 2017. "Open Texture and Schematicity as Arguments for Non-Referential Semantics." In: Meaning, Context, and Methodology, edited by Sarah-Jane Conrad and Klaus Petrus, 13-30. Berlin: DeGruyter. 
Grice, Herbert Paul. 1957. "Meaning.” Philosophical Review 66 (3): 377-88. https://doi.org/10.2307/2182440

Grice, Herbert Paul. 1961. "The Causal Theory of Perception." Proceedings of the Aristotelian Society, Supplementary Volume 35: 121-52.

Hagoort Peter, Giosuè Baggio, and Roel M. Willems. 2009. "Semantic Unification." In The Cognitive Neurosciences, edited by Michael S. Gazzaniga, 819-36. Boston, MA: MIT Press.

Johnson, Hope. 2018. "Should Lab-Grown Meat be labelled as Meat When It's Available for Sale? The Conversation. Available at: https://theconversation.com/should-lab-grown-meat-be-labelled-as-meat-when-its-available-forsale-93129

Kutas, Marta, and Kara D. Federmeier. 2011. "Thirty Years and Counting: Fnding Meaning in the N400 Component of the Event-Related Brain Potential (ERP)." Annual Review of Psychology 62 (1): 621-47. https://doi.org/10.1146/annurev.psych.093008.131123

Kutas, Marta, and Steven A. Hillyard. 1980. "Reading Senseless Sentences: Brain Potentials Reflect Semantic Incongruity." Science 207 (4427): 203-05. https://doi.org/10.1126/science.7350657

Kutas, Marta, and Steven A. Hillyard. 1984. "Brain Potentials during Reading Reflect Word Expectancy and Semantic Association." Nature 307 (5947): 16163. https://doi.org/10.1038/307161a0

Kutas, Marta, Timothy E. Lindamood, and Steven A. Hillyard. 1984. "Word Expectancy and Event-Related Brain Potentials during Sentence Processing." In Preparatory States and Processes, edited by Sylvan Kornblum and J. Renquin, 217-37. Englewood Cliffs, NJ: Erlbaum Press.

Labov, William. 1971. "The Notion of 'System' in Creole Studies." In Pidginization and Creolization of Languages, edited by Dell Hymes, 447-72. Oxford: Cambridge University Press.

Lewis, David. 1969. Convention: A Philosophical Study. Cambridge, MA: Harvard University Press.

Lewis, David. 1979. "Scorekeeping in a Language Game." Journal of Philosophical Logic 8 (3): 339-59. https://doi.org/10.1007/BF00258436

Ludlow, Peter. 2014. Living Words: Meaning Underdetermination and the Dynamic Lexicon. Oxford: Oxford University Press. https://doi.org/10.1093/acprof:oso/9780198712053.001.0001

Noonan, Michael. 1999. "Non-Structuralist Syntax." In Functionalism and Formalism in Linguistics: General Papers, edited by Michael Darnel, 11-33. Philadelphia: John Benjamins Publishing Company.

https://doi.org/10.1075/slcs.41.03noo 
Orlean, Susan. 2011. "The Dog Star." The New Yorker. Available at: https://www.newyorker.com/magazine/2011/08/29/the-dog-star

Peregrin, Jaroslav. 2014a. "Implicit Rules." Organon F 21 (3): 381-98.

Peregrin, Jaroslav. 2014b. Inferentialism: Why Rules Matter. London: Palgrave Macmillan. https://doi.org/10.1057/9781137452962

Pollock, Joanna. 2014. "Mental Content, Holism and Communication." PhD. thesis. University of Edinburgh. Available at: https://www.era.lib.ed.ac.uk/handle/1842/9853

Quine, Willard Van Orman. 1960. Word and Object. Cambridge: MIT Press. Rapaport, William J. 2000. "How to Pass a Turing Test: Syntactic Semantics, Natural Language Understanding, and First-Person Cognition." Journal of Logic, Language, and Information 9 (4): 467-90. https://doi.org/10.1023/A:1008319409770

Rapaport, William J. 2003: "What Did You Mean by That: Misunderstanding, Negotiation, and Syntactic Semantics." Minds and Machines 13 (3): 397-427. https://doi.org/10.1023/A:1024145126190

Recanati, Francois. 2004. Literal Meaning. Cambridge: Cambridge University Press. https://doi.org/10.1017/CBO9780511615382

Recanati, Francois. 2010. "Pragmatic Enrichment." In Routledge Companion to Philosophy of Language, edited by Delia Fara and Gillian Russell, 67-78. New York: Routledge.

Shapiro, Stewart. 2006. Vagueness in Context. Oxford: Clarendon Press. https://doi.org/10.1093/acprof:oso/9780199280391.001.0001

Travis, Charles. 1997. "Pragmatics." In A Companion to the Philosophy of Language, edited by Bob Hale and Crispin Wright, 87-107. Oxford: Blackwell. https://doi.org/10.1002/9781118972090.ch6

Waismann, Friedrich. 1945. "Verifiability." Proceedings of the Aristotelian Society, Supplementary Volumes 19 (1): 119-50.

Weiss, Bernhard. 2010: How to Understand Language. Durham: Acumen. https://doi.org/10.1017/UPO9781844654468

Wernig, Markus, and Erica Cosentino. 2017. "The Interaction of Bayesian Pragmatics and Lexical Semantics in Linguistic Interpretation." In Proceedings of the 39th Annual Conference of the Cognitive Science Society, edited by Glenn Gunzelmann, Andrew Howes, Thora Tenbrink, and Eddy Davelaar, 3504-09. Austin, TX: Cognitive Science Society.

Wilson, Mark. 1982. "Predicate Meets Property." The Philosophical Review 91 (4): 549-89. https://doi.org/10.2307/2184801

Wilson, Mark. 2006. Wandering Significance: An Essay on Conceptual Behavior. Oxford: Oxford University Press. https://doi.org/10.1093/acprof:oso/9780199269259.001.0001 